\title{
ON THE SHARP CONSTANT FOR STARLIKENESS
}

\section{CHEN KEYING}

(Received 24 February 2000)

\begin{abstract}
We obtain a sharp constant of the sufficient condition for $p$-valently starlikeness, which had been studied by Nunokawa (1991), Obradović and Owa (1989), and Li (1993).
\end{abstract}

2000 Mathematics Subject Classification. Primary 30C45.

1. Introduction. Let $A(p)$ denote the class of functions of the form

$$
f(z)=z^{p}+\sum_{n=p+1}^{\infty} a_{n} z^{n} \quad(p \in \mathbb{N})
$$

which are analytic in $\mathbf{U}=\{z:|z|<1\}$. A function $f(z)$ in $A(p)$ is said to be $p$-valently starlike if and only if

$$
\varkappa\left[\frac{z f^{\prime}(z)}{f(z)}\right]>0 \text { in } \mathbf{U} \text {. }
$$

Let $S(p)$ denote the subclass of $A(p)$ consisting of all functions $f(z)$ which are $p$-valently starlike in $\mathbf{U}$ (cf. [1]). For a function $g(z)$ in $A(p)$, the interesting problem is to find the best constant $A$ such that $g(z)$ is in $S(p)$ whenever

$$
\left|1+\frac{z g^{(p+1)}(z)}{g^{(p)}(z)}\right|<A\left|\frac{z g^{(p)}(z)}{g^{(p-1)}(z)}\right| \text { in } \mathbf{U} \text {. }
$$

In 1989, Obradović and Owa [6] obtained that $A=5 / 4$ for the case of $p=1$. For the general case, Nunokawa [5] gained that $A=\log 4$. Recently, Li [2] improved these results and obtained that $A=3 / 2$. In this paper, we will solve this problem completely and give the sharp constant $A=1.80898 \ldots$, where $A$ is the unique solution of the equation

$$
x e^{1 /\left(x^{2}-1\right)}=x+1 .
$$

For proving our result, we should recall the concept of subordination between analytic functions. Given two analytic functions $f(z)$ and $F(z)$, the function $f(z)$ is said to be subordinate to $F(z)$ if $F(z)$ is univalent in $\mathbf{U}, f(0)=F(0)$, and $f(\mathbf{U}) \subset F(\mathbf{U})$. We denote this subordination by $f(z) \prec F(z)$ (see [7]).

Suppose that $h(z)$ is analytic in $\mathbf{U}$, and that $\Phi(z)$ is analytic in an appropriate domain D, we consider the following first-order differential subordination

$$
\beta+z p^{\prime}(z) \Phi(p(z)) \prec h(z),
$$


where $p(z)$ is analytic in $\mathbf{U}, \beta$ is a complex constant. Changing the " $\prec$ " of (1.5) to "=”, we get the corresponding first-order differential equation

$$
\beta+z p^{\prime}(z) \Phi(p(z))=h(z) .
$$

2. Main results. Our results rest on the following lemma, which is the special case of [3, Theorem 3].

LEMMA 2.1. Suppose that $h(z)$ is a starlike function in $\mathrm{U}, \Phi(z)$ is analytic in the domain $\mathbf{D}$ and $p(z), q(z)$ are two analytic functions in $\mathbf{U}$. If $p(z)$ satisfies the relation (1.5), $q(z)$ is a univalent solution of the corresponding equation (1.6) and $p(0)=q(0)$, then $p(z) \prec q(z)$.

THEOREM 2.2. Let $g(z) \in A(p)$, and suppose that

$$
\left|1+\frac{z g^{(p+1)}(z)}{g^{(p)}(z)}\right|<A\left|\frac{z g^{(p)}(z)}{g^{(p-1)}(z)}\right| \text { in } \mathbf{U},
$$

where the constant $A$ is given by (1.4). Then $g(z) \in S(p)$ and the result is sharp.

Proof. Let

$$
f(z)=\frac{g^{(p-1)}(z)}{p !} .
$$

Then $f(z) \in A(1)$. From the assumption (2.1), $f(z)$ satisfies

$$
\left|1+\frac{z f^{\prime \prime}(z)}{f^{\prime}(z)}\right|<A\left|\frac{z f^{\prime}(z)}{f(z)}\right| \text { in } \mathbf{U} .
$$

By putting $p(z)=z f^{\prime}(z) / f(z)$, equation (2.3) can be rewritten as

$$
\left|1+\frac{z p^{\prime}(z)}{p^{2}(z)}\right|<A
$$

Let $\varphi(z)=A(1+A z) /(A+z)$ for $z \in \mathbf{U}$. Obviously $\varphi(z)$ is a conformal mapping from $\mathbf{U}$ to $\Omega=\{w:|w|<A\}$ and $\varphi(0)=1$. Combining (2.4) with the definition of subordination, we obtain

$$
1+\frac{z p^{\prime}(z)}{p^{2}(z)} \prec \frac{A(1+A z)}{A+z} .
$$

Setting

$$
q(z)=\frac{1}{1+\left(A^{2}-1\right) \log A /(A+z)}
$$

we have

$$
1+\frac{z q^{\prime}(z)}{q^{2}(z)}=\frac{A(1+A z)}{A+z}
$$

and $p(0)=q(0)=1$. As $A>1$, we can choose a uniform analytic branch of $\log (A+z)$ such that $q(z)$ is univalent on this branch. By taking the real part of the denominator of $q(z)$ and combining (1.4), we conclude that

$$
\mathfrak{\gtrless}\left[1+\left(A^{2}-1\right) \log \frac{A}{A+z}\right]>1+\left(A^{2}-1\right) \log \frac{A}{A+1}=0 .
$$


It follows that $\mathfrak{R}[q(z)]>0$, so $q(z)$ is analytic and univalent. Let $\mathbf{D}=\mathbb{C} \backslash\{0\}$, $\Phi(z)=1 / z^{2}, \beta=1$, and $h(z)=A(1+A z) /(A+z)$, where $\mathbb{C}$ is the complex plane. It is clear that $h(z)$ is a starlike function. From Lemma 2.1, we deduce that $p(z) \prec q(z)$. Hence

$$
\mathfrak{\gtrless}\left[\frac{z f^{\prime}(z)}{f(z)}\right]=\mathfrak{\Re}[p(z)] \geq \min _{|z|=r<1} \mathfrak{\Re}[q(z)]>0 .
$$

This is equivalent to

$$
\mathfrak{\gtrless}\left[\frac{z g^{(p)}(z)}{\mathfrak{g}^{(p-1)}(z)}\right]=\mathfrak{\gtrless}\left[\frac{z f^{\prime}(z)}{f(z)}\right]>0 \text { in } \mathbf{U} .
$$

From [4, Theorem 5], we have

$$
\mathfrak{\gtrless}\left[\frac{z g^{\prime}(z)}{g(z)}\right]>0 \text { in } \mathbf{U}
$$

This proves $g(z) \in S(p)$.

For any $A_{1}>A=1.80898 \ldots$, we get a function $q_{1}(z)$ by replacing $A$ in (2.6) with $A_{1}$ and choosing an appropriate branch of $\log \left(A_{1}+z\right)$. We can easily observe that the real part of $q_{1}(z)$ is not always positive. Through the relations $q_{1}(z)=z f^{\prime}(z) / f(z)$ and $f(z)=g^{(p-1)}(z) / p$ !, we can construct an analytic function $g(z)$ which belongs to $A(p)$ and satisfies (2.1), but it is not in $S(p)$. This completes the proof.

Taking $p=1$ in Theorem 2.2, we easily have the following corollary.

COROLLARY 2.3. If $f(z) \in A(1)$ and it satisfies the condition

$$
\left|1+\frac{z f^{\prime \prime}(z)}{f^{\prime}(z)}\right|<A\left|\frac{z f^{\prime}(z)}{f(z)}\right| \text { in } \mathbf{U},
$$

where the constant $A$ is given by (1.4), then $f(z)$ is univalent and starlike in $\mathbf{U}$.

The problem that Nunokawa proposed in [5] has been solved completely, but the converse proposition of Theorem 2.2 is not true. We find a simple example $f(z)=$ $z /(1-z)$ which belongs to $S(1)$, but it does not satisfy (2.12). The following theorem is better than (2.1) because it includes at least this example.

THEOREM 2.4. Let $g(z) \in A(p)$, and suppose that

$$
\left|1+\frac{z g^{(p+1)}(z)}{g^{(p)}(z)}-\frac{z g^{(p)}(z)}{\mathcal{g}^{(p-1)}(z)}\right|<\left|\frac{z \mathcal{g}^{(p)}(z)}{\mathcal{g}^{(p-1)}(z)}\right| \text { in } \mathbf{U} \text {. }
$$

Then $g(z) \in S(p)$.

Proof. Let

$$
f(z)=\frac{g^{(p-1)}(z)}{p !} .
$$

Then $f(z) \in A(1)$. From the assumption (2.13), $f(z)$ satisfies

$$
\left|1+\frac{z f^{\prime \prime}(z)}{f^{\prime}(z)}-\frac{z f^{\prime}(z)}{f(z)}\right|<\left|\frac{z f^{\prime}(z)}{f(z)}\right| \text { in } \mathbf{U} \text {. }
$$


By setting $p(z)=z f^{\prime}(z) / f(z)$, equation (2.15) can be rewritten as

$$
\left|\frac{z p^{\prime}(z)}{p^{2}(z)}\right|<1 .
$$

From the definition of subordination, we obtain

$$
\frac{z p^{\prime}(z)}{p^{2}(z)} \prec z
$$

Let $q(z)=1 /(1-z)$, we observe that $z q^{\prime}(z) / q^{2}(z)=z, p(0)=q(0)=1$, and $\mathfrak{z}[q(z)]$ $>0$. From Lemma 2.1, we know that $p(z) \prec 1 /(1-z)$. Therefore

$$
\mathfrak{\gtrless}\left[\frac{z f^{\prime}(z)}{f(z)}\right]=\mathfrak{\Re}[p(z)] \geq \min _{|z|=r<1} \mathfrak{\Re}[q(z)]>0 .
$$

This is equivalent to

$$
\mathfrak{\varkappa}\left[\frac{z g^{(p)}(z)}{\mathcal{g}^{(p-1)}(z)}\right]=\mathfrak{\gtrless}\left[\frac{z f^{\prime}(z)}{f(z)}\right]>0 \text { in } \mathbf{U} .
$$

From [4, Theorem 5], we have

$$
\mathfrak{\gtrless}\left[\frac{z g^{\prime}(z)}{g(z)}\right]>0 \quad \text { in } \mathbf{U} .
$$

This completes the proof.

Taking $p=1$ in Theorem 2.4, we obviously have the following corollary.

COROLLARY 2.5. If $f(z) \in A(1)$ and it satisfies the condition

$$
\left|1+\frac{z f^{\prime \prime}(z)}{f^{\prime}(z)}-\frac{z f^{\prime}(z)}{f(z)}\right|<\left|\frac{z f^{\prime}(z)}{f(z)}\right| \text { in } \mathbf{U},
$$

then $f(z) \in S(1)$.

ACKNOWLedgements. I wish to express my gratitude to Professor Hu Ke and Professor Fang Ainong for their guidance, advice, and encouragement in my work, past and present. I am also grateful to the referee for his valuable advice.

This research was supported by China NSF (Grant No. 19531060) and Doctor Spot Foundation (Grant No. 97024811).

\section{REFERENCES}

[1] A. W. Goodman, On the Schwarz-Christoffel transformation and $p$-valent functions, Trans. Amer. Math. Soc. 68 (1950), 204-223. MR 11,508d. Zbl 037.05502.

[2] J. L. Li, On a criterion of starlikeness, Math. Japon. 38 (1993), no. 5, 897-899. MR 94j:30012. Zbl 786.30010.

[3] S. S. Miller and P. T. Mocanu, On some classes of first-order differential subordinations, Michigan Math. J. 32 (1985), no. 2, 185-195. MR 86h:30046. Zbl 575.30019.

[4] M. Nunokawa, On the theory of multivalent functions, Tsukuba J. Math. 11 (1987), no. 2, 273-286. MR 89d:30013. Zbl 639.30014. 
[5] — On certain multivalent functions, Math. Japon. 36 (1991), no. 1, 67-70. MR 92b:30017. Zbl 718.30010.

[6] M. Obradović and S. Owa, A criterion for starlikeness, Math. Nachr. 140 (1989), 97-102. MR 90i:30020. Zbl 676.30009.

[7] C. Pommerenke, Univalent Functions. With a chapter on quadratic differentials by Gerd Jensen, Studia Mathematica/Mathematische Lehrbücher, vol. 25, Vandenhoeck \& Ruprecht, Göttingen, 1975. MR 58\#22526. Zbl 298.30014.

Chen Keying: Department of ApPlied Mathematics, Shanghai Jiaotong University, SHANGHAI 200240, CHINA

E-mail address: kychen801@mai11.sjtu.edu.cn 


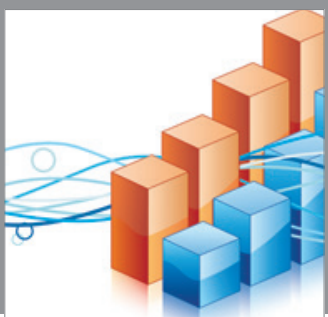

Advances in

Operations Research

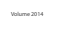

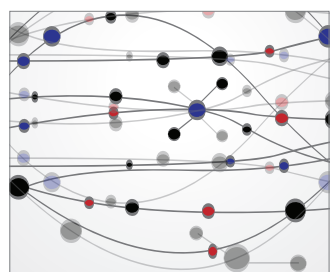

\section{The Scientific} World Journal
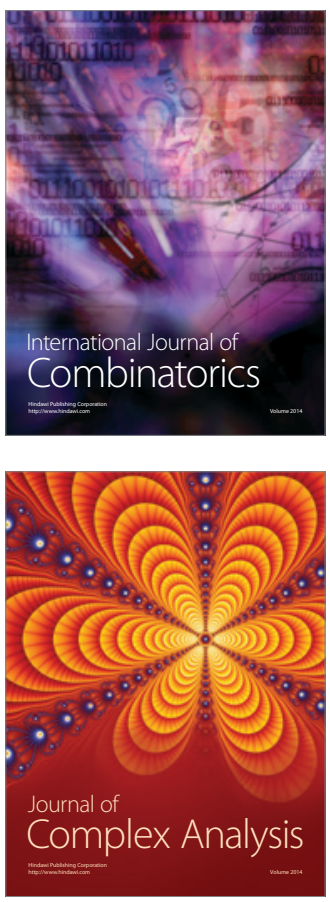

International Journal of

Mathematics and

Mathematical

Sciences
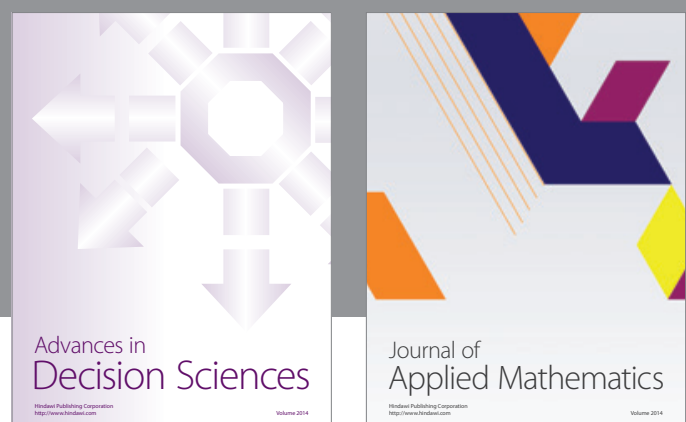

Journal of

Applied Mathematics
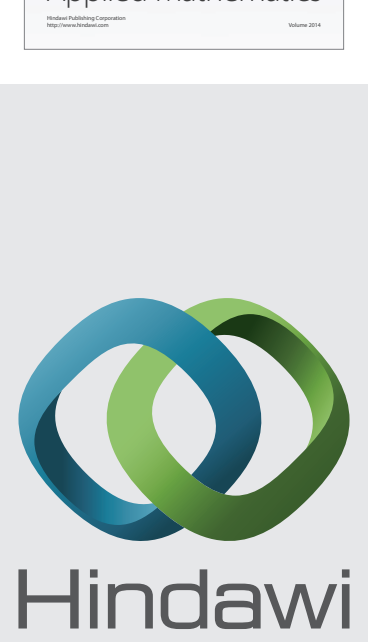

Submit your manuscripts at http://www.hindawi.com
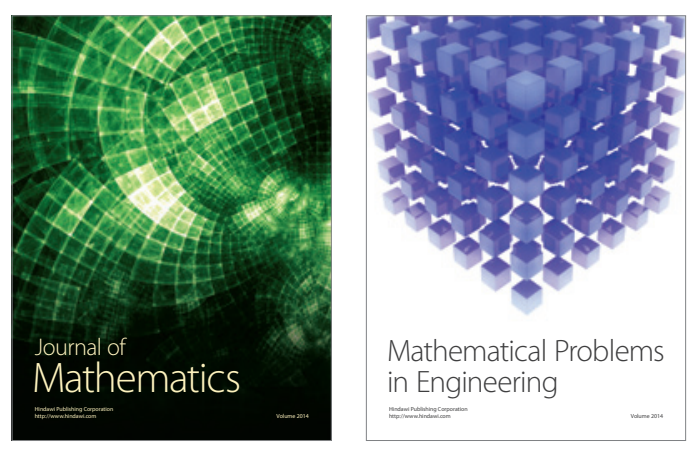

Mathematical Problems in Engineering
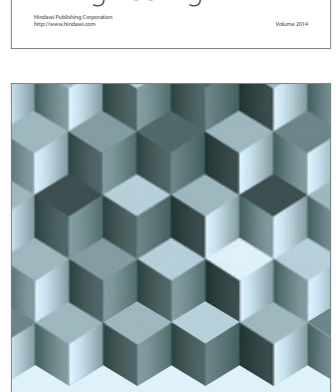

Journal of

Function Spaces
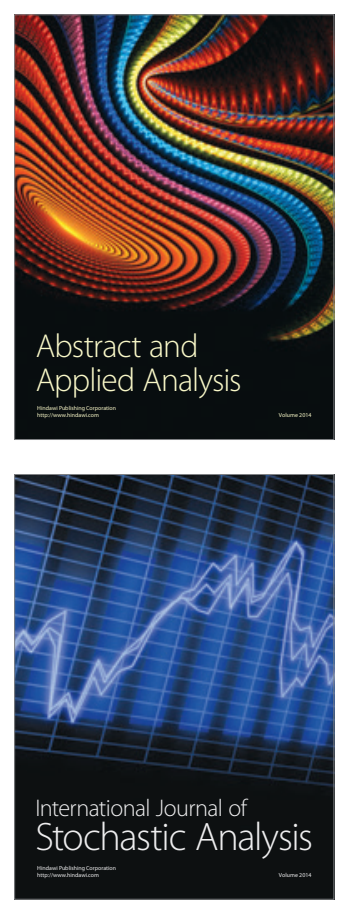

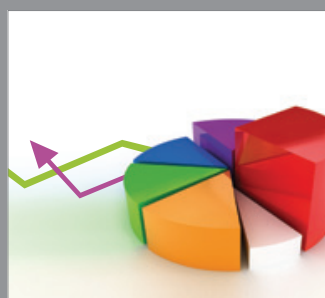

ournal of

Probability and Statistics

Promensencen
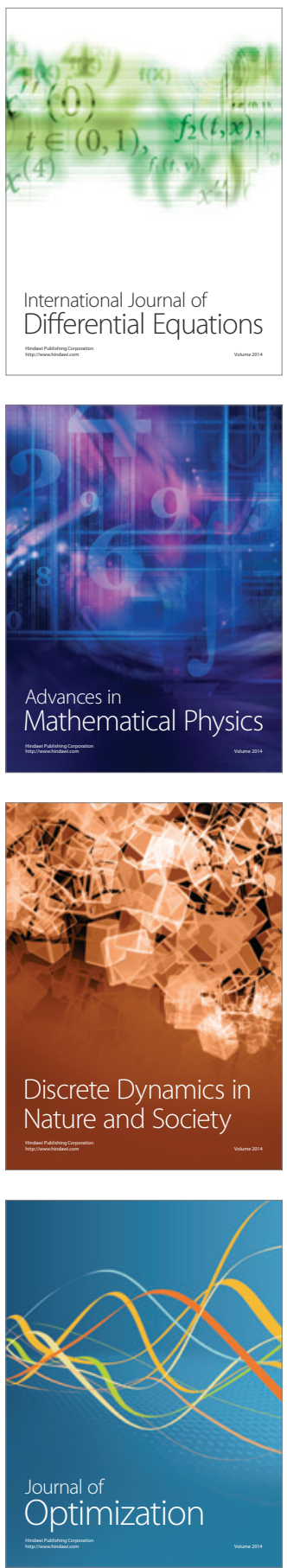